\title{
Socio-Technical Evaluation Matrix (STEM): A Collaborative Tool to Support and Facilitate Discussion on Socio-Technical Issues of a Design Process
}

\author{
Souleymane Boundaouda Camara and José Abdelnour-Nocera \\ Thames Valley Univeristy, St Mary's Road, Ealing, London, W5 2RF, UK \\ souleymane.camara@tvu.ac.uk
}

\begin{abstract}
STEM is an interactive web application designed to support and facilitate socio-technical discussions in a collaborative environment. This interactive demo shows how STEM aims to encompass issues with collaborative tools such as organisation of discussion threads by relevancy, interdependency and iteration of previous discussions.
\end{abstract}

Keywords: Collaborative work tool, socio-technical evaluation, dependability.

\section{STEM: Overview}

$\mathrm{STEM}^{1}$ is an interactive web application designed to support and facilitate collaboration in IT system design where, social and technical factors need to be matched to identify critical issues towards system acceptance, use and fitness for purpose. Emerging from an ongoing research in the Village eScience for Life $\left(\mathrm{VeSeL}^{2}\right)$ project, this tool tries to offer an improved environment for group discussion based on pre-defined framework, methods or models.

The demonstration of the tool will highlight the rational for STEM and an interactive online session of its main features based on its usage in the VeSeL project as described in the following sections.

\section{Why STEM: Features and Rational}

STEM builds on a rationalised approach and philosophy of understanding the use of HCI moderated collaborative work which offers:

- Distinction of Critical Social and Technical Factors of System Design/Evaluation. Socio-technical evaluation highlights the importance and dependability of a system based the social impact of actors (users, designers) and the technological impact. STEM allows users to comment on the implication of design decisions for both users and the technology. A decision that

\footnotetext{
${ }^{1}$ STEM overview available at: http://itcentre.tvu.ac.uk/ VeSeL/matrixsample/.

${ }^{2}$ Visit http://www.lkl.ac.uk/projects/vesel/ for more on the VeSeL project.
} 
is expressed for one is therefore evaluated in its implication for the other in a form based interface.

- Adaptability to Pre-Defined Design Frameworks and Methodologies. Most collaborative tools, open source ${ }^{3}$ or proprietary (Lotus Notes ${ }^{4}$, Freeman ${ }^{5}$, Huddle $^{6}$ ) are often too generic with no underpinning HCI framework or methodologies. They aim to manage workflows, documents and communications within a group. With STEM, users propose dimensions and relevant attributes of an approach to adopt and evaluate their processes against them. The demonstration will show how these dimensions and attributes can be defined.

- Better Organisation of Discussion Thread: Many collaborative tools tend to display discussion entries in a chronological manner or simply alter previous entries as the discussions feed in new or better ideas (Google docs, Wikipedia). Following a topic thread on these tools is therefore very difficult. STEM interface allows participants to decide if their contribution is in support or conflicts with an existing main entry. It then displays the comments by relevance and in relation to each other saving time and motivating participants to easily follow/contribute to a discussion. Furthermore, a moderator of a discussion can summarise the discussion by attributes if there are too many or sufficient entries.

- Iterative Capability for Consistent and Continuous Design Processes: Often in IT system design, processes follow an iterative route. Requirements are gathered, refined and turned into a design prototype. Then, prototypes are discussed, summarised for evaluation and so on. STEM goes a step further by integrating the summary of previous stages into the discussion of the actual stage. The reason for this is that some dimensions may not or can not be addressed in previous stages. By bringing them forward, STEM maximises the chance of them being acted upon at the earliest possibility.

These features are currently being evaluated to refine the next version of the tool.

\section{Conclusion}

STEM is not a platform for technicality elucidation, document exchange or a heavy communication-driven collaborative tool. Rather, it is an environment for discussing cross-party issues on a predefined approach. It is an attempt to address the shortcomings of current collaborative tools in HCI based design.

\footnotetext{
${ }^{3}$ Open source tools: http://php.opensourcecms.com/scripts/show.php?catid=4\&cat=Groupware

${ }^{4}$ Lotus Notes by IBM: http://www-01.ibm.com/software/lotus/

${ }^{5}$ Freeman Online: http://www.freemanco.com/showorganizers/collaborativeTools.jsp

${ }^{6}$ Huddle: http://www.huddle.net/what-is-huddle
} 